\title{
Oxidized LDL as A Biomarker in Metabolic Syndrome
}

Wahid Ali", Umesh Prasad Kushwaha, Mohd Wamique, Preeti Vishwakarma, Mohd Tasleem, Mabood Khan and Ashustosh Kumar

Department of Pathology, King George's Medical University, Lucknow, U.P., India

*Corresponding author: Wahid Ali, Department of Pathology, King George's Medical University, Lucknow, U.P., India, Tel: +91-9198852343; E-mail: aliwahid78@gmail.com

Received date: August 08, 2017; Accepted date: September 25, 2017; Published date: September 30, 2017

Copyright: ( 2017 Ali W, et al. This is an open-access article distributed under the terms of the Creative Commons Attribution License, which permits unrestricted use, distribution, and reproduction in any medium, provided the original author and source are credited.

\section{Abstract \\ Objective: To study the correlation of lipids and lipoprotein indices with special reference to oxidized LDL in study subjects.}

Material and Methods: Study comprised of total 124 subjects. 104 symptomatic cases either sex age between 20-80 years were recruited, of which, 10 were type-2 diabetes mellitus(Type- 2DM), 63 were with metabolic syndrome (MS), and 31 were type-2 diabetes mellitus with hypertension (Type-2DM+HT). Total 20, age and sex matched normal healthy subjects were recruited and served as control group for the present study.

Results: Comparing the mean OxLDL of four groups (Type-2DM, MS, Type-2DM+HT and normal), by one way ANOVA showed significantly $(p<0.001)$ differences among the groups. Further, Bonferroni test showed that the mean OxLDL rose significantly $(p<0.001)$ in cases (Type-2DM, MS and Type-2DM+HT) groups as compared to normal group but no differences were observed among the cases.

Conlusion: It is concluded that OxLDL may serves as better predictive biomarker in metabolic syndrome individuals.

Keywords: Ox -LDL; HDL; VLDL; Diabetes mellitus; Metabolic Syndrome

\section{Introduction}

Visceral Obesity, Insulin resistance, Dyslipidaemia, Hypertension and pro-inflammatory or thrombotic state collectively define metabolic syndrome [1-2]. Metabolic syndrome is a combination of unfavorable health factors including abdominal obesity, dyslipidemia, hypertension and glucose intolerance and is strongly associated with increased risk of cardiovascular disease (CVD) and type-2 diabetes [3-4]. One of the key drivers in the development of metabolic syndrome is obesity [5]. Oxidized LDL, a marker of lipoproteinassociated Oxidative stress, is an emerging cardiovascular risk factor Oxidized LDL activates circulating monocytes, thereby increasing their ability to infiltrate the Vascular wall. This increased infiltration is a primary stage in atherogenesis [6]. Oxidative stress (OX) is known to play a major role in the pathogenesis of Metabolic Syndrome.Oxidative stress means an imbalance between production of reactive oxygen species and antioxidant defence system that buffers the oxidative damage, and is considered to be one of the main causes of molecular damage to cellular and tissue structures $[7,8]$. This imbalance in the rate at which the intracellular content of free radicals, produced through a number of cellular events, increases relative to the capacity of the cell to dispose of these oxidants, when not neutralized, these free radicals have the capacity to alter the integrity of numerous molecules such as lipids, proteins and DNA. Lipids are a major target of free radical attack, which induces lipid peroxidation. Free radical induced peroxidation of membrane lipids is critical for memebrane damage. Because it leads to alterations in the biophysical properties of the membrane, such as the degree of fluidity, and can lead to inactivation of membrane-bound receptors or enzymes, which in turn may impair normal cellular function. Therefore, measurement of products of lipid peroxidation has been commonly used to assess oxidative stress/injury [9].

Presence of oxidized low-density lipoprotein (OxLDL) is another aspect of oxidative modification. The concentration of circulating oxidized LDL (OxLDL) has shown relationships with atherosclerotic disease, and some studies indicate OxLDL concentrations to be increased in the metabolic syndrome. Although the origin of OxLDL is unknown, the vascular wall is considered the main source. However, the oxidative stress status in patients with Metabolic Syndrome remains unclear. The challenge is to identify high risk individuals as through effective identification and intervention of risk factors, so that the disease can be halted successfully [10]. One strategy is to understand the overlying pathogenesis and disease mechanisms which will possibly help in identifying surrogate markers to effectively evaluate the overall risk of these individuals.

\section{Materials and Methods}

The present study is a cross sectional study, carried out at Department of Pathology, King Georg's Medical University, Lucknow from August 2015-August 2016. Human peripheral blood samples were collected from Department of Cardiology OPD as well as from Department of Medicine. The present study determines association of lipids and lipoproteins indices with special interest to oxidized low density lipoprotein in metabolic syndrome. This study comprised of total 124 subjects. 104 symptomatic cases either sex age between 20-80 years were recruited, of which 10 were type- 2 diabetes mellitus (Type2DM), 63 were with metabolic syndrome (MS), and 31 were type-2 
diabetes mellitus with hypertension (Type-2DM+HT). 20 age and sex matched normal healthy subjects were served as control group. Lipid profile of the subjects was classified based on ATP III model [11]. Patients are recruited after obtaining their written informed consent. Ethical clearance was obtained for the study from the Institutional Ethics Committee. (Ethical clearance no: 71 ECM II B Thesis/P 13).

\section{Biochemical investigations}

Total $5 \mathrm{ml}$ of the fasting peripheral blood was collected from patient as well as control into the plain serum vial and allowed to clot. The blood was centrifuged at $3000 \mathrm{rpm}$ for $15 \mathrm{~min}$ at room temperature. Serum was separated in $2 \mathrm{ml}$ micro centrifuge tube and store in $-80^{\circ} \mathrm{C}$ until further analysis .Serum triglyceride was estimated by enzymaticcolorimetric end point method. HDL cholesterol was estimated by coupled classic precipitation method using diazyme's HDL-cholesterol kit. VLDL and LDL cholesterol was calculated by Friedwald's formulae considering its limitations. Blood glucose was estimated by enzymaticcolorimetric, trinder-kinetic. All the parameters which were under investigation were determined in the serum of subjects by using commercially available reagents kits. In vitro quantitative determination of Human oxidized low density lipoprotein (OxLDL) concentrations in serum was done by Elabscience ELISA Kit recognizes natural and recombinant Human OxLDL.

\section{Human oxidized low density lipoprotein ELISA}

Human oxidized low density lipoprotein was estimated using ELISA kit based on sandwich-ELISA method. The micro ELISA plate provided in the kit is pre-coated with an antibody specific to OxLDL. Standards or samples was added to the appropriate micro ELISA plate wells and combined with the specific detection antibody. Then a biotinylated detection antibody specific for OxLDL and AvidinHorseradish Peroxidase (HRP) conjugate was added to each micro plate well successively and incubated. Free components were washed away. The substrate solution was added to each well. Only those wells that contained OxLDL, was observed blue in color due to biotinylated detection antibody and Avidin-Horseradish Peroxidase (HRP) conjugation. The enzyme-substrate reaction was terminated by the addition of a sulphuric acid solution which turned blue color to yellow. The optical density (OD) was measured spectrophotometrically at a wavelength of $450 \mathrm{~nm} \pm 2 \mathrm{~nm}$. The OD value was proportional to the concentration of OxLDL. Concentration of OxLDL was calculated in the samples by comparing the $\mathrm{OD}$ of the samples to the standard curve.

\section{Statistical analysis}

The results are presented as Mean \pm Standard error of the mean (SEM) using SPSS statistical computer package version 10 software (SPSS 10, 1999). Student's t-test was used to ascertain the significance of the difference between two means of continuous variables. ANOVA is used for difference in mean values of more than two grouped data. Bonferroni multiple comparison tests were used to calculate differences between group means. $\mathrm{P}<0.05$ was considered as statistically significant.

\section{Results}

The age of healthy controls, Type-2DM, MS and Type-2DM+HT ranged from 32-70 yrs, 35-64 yrs, 20-78 yrs and 28-80 yrs respectively with mean $( \pm$ SE) $54.25 \pm 2.45 \mathrm{yrs}, 48.80 \pm 3.23 \mathrm{yrs}, 46.56 \pm 1.83 \mathrm{yrs}$ and $53.03 \pm 2.28$ yrs respectively and median $54 \mathrm{yr}, 49 \mathrm{yrs}, 45 \mathrm{yrs}$ and $50 \mathrm{yrs}$, respectively. The mean age of normal group was the highest and MS the least. Comparing the mean age of four groups showed similar age among the groups $(\mathrm{F}=2.62, \mathrm{p}=0.054)$ i.e. did not differed significantly. Further, there were $8(40.0 \%)$ females and $12(60.0 \%)$ males in Normal group while it were $2(20.0 \%)$ and $8(80.0 \%)$ respectively in Type-2DM group, 32 (50.8\%) and 31 (49.2\%) respectively in MS group, and 19 (61.3\%) and 12 (38.7\%) respectively in Type $-2 \mathrm{DM}+\mathrm{HT}$ group. In all groups, the frequency (\%) of males was higher than females except MS group. The OxLDL of four groups showed significantly $(\mathrm{p}<0.001)$ different mean OxLDL among the groups (Table 1). Further, Bonferroni test showed that the mean OxLDL rose significantly $(\mathrm{p}<0.001)$ in cases (Type-2DM, MS and Type-2DM+HT) groups as compared to Normal group (Table 2) but not differs among the cases i.e. found to be statistically the same.

\begin{tabular}{|l|l|l|l|l|l|l|}
\hline Variables & Normal $(\mathbf{n = 2 0})$ & Type-2DM $(\mathbf{n}=\mathbf{1 0})$ & MS $(\mathbf{n}=\mathbf{6 3})$ & Type- 2DM+HT $(\mathbf{n}=\mathbf{3 1})$ & F value & $\mathbf{p}$ value \\
\hline OxLDL $(\mathrm{ng} / \mathrm{mL})$ & $108.36 \pm 3.94$ & $195.87 \pm 17.30$ & $200.24 \pm 6.36$ & $213.36 \pm 7.71$ & 25.49 & $<0.001$ \\
\hline
\end{tabular}

Table 1: OxLDL (Mean \pm SE) of four groups.

\begin{tabular}{|l|l|}
\hline Comparisons & OxLDL \\
\hline Normal vs. Type-2DM & $<0.001$ \\
\hline Normal vs. MS & $<0.001$ \\
\hline Normal vs. Type-2DM+HT & $<0.001$ \\
\hline Type-2DM vs. MS & 1 \\
\hline Type-2DM vs. Type-2DM+HT & 1 \\
\hline MS vs. Type-2DM+HT & 1 \\
\hline
\end{tabular}

Moreover, OxLDL also showed significant diagnostic importance when evaluated between normal and total cases (Type-2DM + MS + Type-2DM+HT) and at cut off value of $>135.6$ it is discriminating the subjects of two groups significantly ( $\mathrm{AUC}=0.954, \mathrm{Z}=25.82, \mathrm{p}<0.001$ ) with high $90.38 \%$ sensitivity $(95 \% \quad C I=83.0-95.3)$ and $100.00 \%$ specificity (95\% CI=83.0-100.0) and $100.0 \%$ positive predictive value and $96.7 \%$ negative predictive value. (Table $3 \&$ Figures $1-4$ ).

Table 2: Comparison ( $p$ value) of mean difference in OxLDL between the groups by Bonferroni test. 
Citation: Ali W, Kushwaha UP, Wamique M, Vishwakarma P, Tasleem M, et al. (2017) Oxidized LDL as A Biomarker in Metabolic Syndrome. J

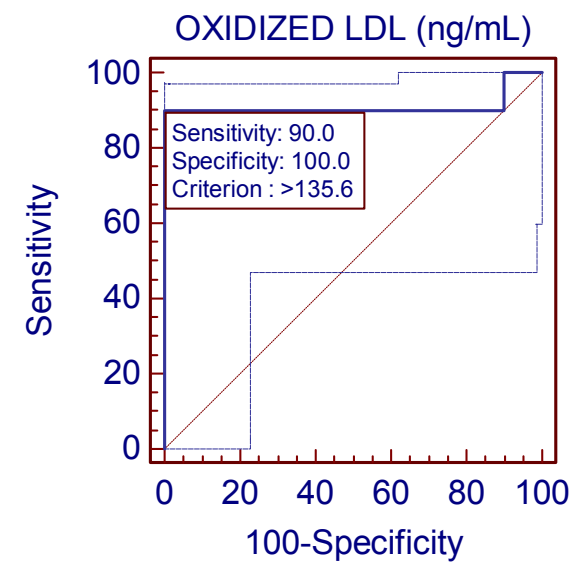

Figure 1: Diagnostic accuracy of OxLDL to discriminate normal and Type-2DM cases.

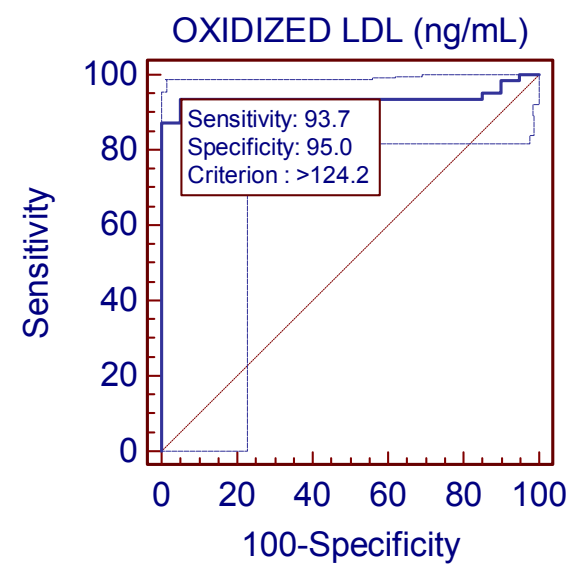

Figure 2: Diagnostic accuracy of OxLDL to discriminate normal and metabolic syndrome cases.

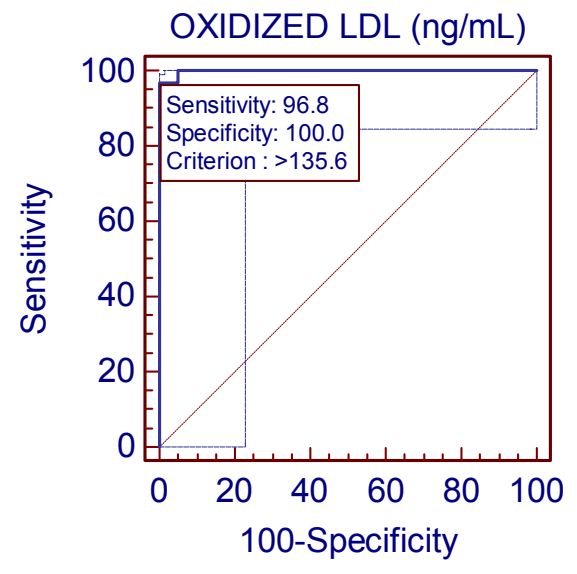

Figure 3: Diagnostic accuracy of OxLDL to discriminate normal and Type-2DM $+\mathrm{HT}$ cases.

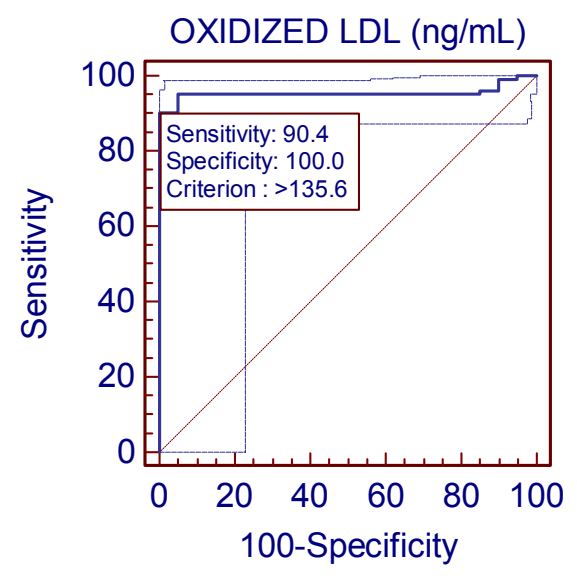

Figure 4: Diagnostic accuracy of OxLDL to discriminate normal and cases (Type-2DM + MS + Type-2DM with HT).

\begin{tabular}{|c|c|c|c|c|c|c|c|c|}
\hline $\begin{array}{l}\text { ROC } \\
\text { Normal vs. }\end{array}$ & $\begin{array}{l}\text { Cut off value } \\
\text { (ng/ml) }\end{array}$ & AUC & $Z$ value & $P$ value & $\begin{array}{l}\text { Sensitivity } \\
(95 \% \mathrm{Cl})\end{array}$ & $\begin{array}{l}\text { Specificity } \\
(95 \% \text { Cl) }\end{array}$ & $+P V$ & $-P V$ \\
\hline Type-2 DM & $>135.6$ & 0.91 & 6.17 & $<0.001$ & $90.00(55.5-98.3)$ & $100.00(83.0-100.0)$ & 100 & 95.2 \\
\hline MS & $>124.2$ & 0.94 & 17.69 & $<0.001$ & $93.65(84.5-98.2)$ & $95.00(75.1-99.2)$ & 98.3 & 82.6 \\
\hline Type-2DM +HT & $>135.6$ & 0.998 & 95.25 & $<0.001$ & $96.77(83.2-99.5)$ & $100.00(83.0-100.0)$ & 100 & 95.2 \\
\hline Total & $>135.6$ & 0.954 & 25.82 & $<0.001$ & $90.38(83.0-95.3)$ & $100.00(83.0-100.0)$ & 100 & 96.7 \\
\hline
\end{tabular}

Table 3: Diagnostic accuracy of OxLDL to discriminate normal and cases.

The lipid profile of four groups showed significantly $(\mathrm{p}<0.001)$ different mean fasting TG and fasting HDL among the groups (Table
4). Further, Bonferroni test showed that the mean fasting TG raised while fasting HDL decrease significantly $(\mathrm{p}<0.01$ or $\mathrm{p}<0.001)$ in cases 
Page 4 of 5

(Type-2DM, MS and Type-2DM+HT) as compared to Normal group but not differed ( $p>0.05)$ among the cases i.e. found to be statistically the same (Table 5). However, fasting TC and fasting LDL showed increasing trend with severity (Type-2DM $<\mathrm{MS}<$ Type-2DM+HT) but did not reach statistical significance $(p>0.05)$ i.e. found to be insignificant among the groups (Table 6, Figure 2).

\begin{tabular}{|c|c|c|c|c|c|c|}
\hline Variables & $\begin{array}{l}\text { Normal } \\
(n=20)\end{array}$ & $\begin{array}{l}\text { Type-2DM } \\
(n=10)\end{array}$ & $\begin{array}{l}\text { MS } \\
(n=63)\end{array}$ & $\begin{array}{l}\text { Type- 2DM+HT } \\
(n=31)\end{array}$ & $F$ value & $P$ value \\
\hline $\mathrm{TC}(\mathrm{mg} / \mathrm{dL})$ & $151.24 \pm 4.96$ & $167.31 \pm 16.75$ & $170.10 \pm 7.73$ & $175.68 \pm 12.19$ & 0.76 & 0.518 \\
\hline Fasting TG (mg/dL) & $107.54 \pm 8.45$ & $245.83 \pm 21.50$ & $210.15 \pm 9.58$ & $182.31 \pm 14.33$ & 11.23 & $<0.001$ \\
\hline Fasting LDL (mg/dL) & $81.40 \pm 4.13$ & $84.80 \pm 14.56$ & $91.63 \pm 6.31$ & $93.86 \pm 8.03$ & 0.4 & 0.757 \\
\hline Fasting HDL (mg/dL) & $51.73 \pm 1.00$ & $29.23 \pm 3.62$ & $33.67 \pm 2.00$ & $36.55 \pm 3.50$ & 7.97 & $<0.001$ \\
\hline
\end{tabular}

Table 4: Lipid profile (Mean \pm SE) of four groups.

\begin{tabular}{|l|l|l|}
\hline Comparisons & Fasting TG & Fasting HDL \\
\hline Normal vs. Type 2DM & $<0.001$ & 0.001 \\
\hline Normal vs. MS & $<0.011$ & $<0.001$ \\
\hline Normal vs. Type 2DM+HT & 0.002 & 0.005 \\
\hline Type 2DM vs. MS & 0.42 & 1 \\
\hline Type 2DM vs. Type 2DM+HT & 0.099 & 1 \\
\hline MS vs. Type 2DM+HT & 1 & 1 \\
\hline
\end{tabular}

The FBG of four groups showed significantly $(p<0.001)$ different mean FBG among the groups (Table 6). Further, Bonferroni test showed that the mean fasting FBG elevated significantly $(\mathrm{p}<0.001)$ in all case groups (Type-2DM, MS and Type-2DM+HT) as compared to Normal group (Table 7). Conversely, the mean FBG also found elevated significantly $(\mathrm{p}<0.001)$ in Type-2DM and Type-2DM+HT as compared to MS group but not differ $(\mathrm{p}>0.05)$ between Type-2DM and Type-2DM+HT i.e. found to be statistically the same.

Table 5: For each lipid profile, comparison ( $\mathrm{p}$ value) of mean difference between the groups by Bonferroni test.

\begin{tabular}{|l|l|l|l|l|l|l|}
\hline Variables & $\begin{array}{l}\text { Normal } \\
(\mathbf{n = 2 0})\end{array}$ & $\begin{array}{l}\text { Type-2DM } \\
(\mathbf{n = 1 0 )}\end{array}$ & $\begin{array}{l}\text { MS } \\
(\mathbf{n = 6 3 )}\end{array}$ & $\begin{array}{l}\text { Type- 2DM+HT } \\
(\mathbf{n = 3 1})\end{array}$ & $\mathbf{F}$ value & P value \\
\hline FBG $(\mathrm{mg} / \mathrm{dL})$ & $91.76 \pm 2.72$ & $167.18 \pm 15.83$ & $126.44 \pm 3.61$ & $157.52 \pm 5.75$ & 24.99 \\
\hline
\end{tabular}

Table 6: FBG (Mean \pm SE) of four groups.

\begin{tabular}{|l|l|}
\hline Comparisons & FBG \\
\hline Normal vs. Type 2DM & $<0.001$ \\
\hline Normal vs. MS & $<0.001$ \\
\hline Normal vs. Type 2DM+HT & $<0.001$ \\
\hline Type 2DM vs. MS & 0.001 \\
\hline Type 2DM vs. Type 2DM+HT & 1 \\
\hline MS vs. Type 2DM+HT & $<0.001$ \\
\hline
\end{tabular}

fasting total cholesterol, fasting LDL-C and fasting HDL-C) which were associated with metabolic syndrome, type- 2 diabetes and its components. Comparing the mean lipid profile of all four groups showed significantly $(\mathrm{P}<0.001)$ different, mean fasting triglycerides and fasting high-density lipoprotein among the groups. We evaluated that the mean fasting triglycerides was found to be elevated while levels of fasting high-density lipoprotein cholesterol decreases significantly in all the disease groups when compared to the normal group but not significant among the disease groups which was found to be statistically similar. Total fasting cholesterol and fasting lowdensity lipoproteins showed increasing trend with all groups but did not reaches up to the statistically significance level. In our study we also determined that low levels of high-density lipoproteins cholesterol (HDL-C) and elevated levels of triglycerides (TG) in all the groups as compared to the normal group were associated with the risk of metabolic syndrome and type-2 diabetes [12-14].

The association between metabolic syndrome, type- 2 diabetes and its components with fasting blood in glucose level, in Indian subjects in more than 20 years of old patients of metabolic syndrome and type-2 diabetes, was more pronounced. In present study the mean FBG for all four groups showed significant association among all four groups. We have also evaluated that the mean of FBG levels was found 
Page 5 of 5

to be elevated significantly $(<0.001)$ in type- 2 diabetes, metabolic syndrome and type-2 diabetes with hypertension groups when compared to the normal group [15]. In this study we measured the concentration of circulatory oxidized low- density lipoprotein with metabolic syndrome, type- 2 diabetes as well as normal subjects group. Comparing the mean of OxLDL levels in all four groups and found to be significantly $(\mathrm{P}<0.001)$ different among the groups. We also evaluated that the mean OxLDL level elevated significantly $(\mathrm{P}<0.001)$ in disease groups as compared to normal group. Oxidized LDL is a marker of oxidative stress. Increased oxidative stress and inflammatory biomarkers are known to play an important role in the initiation and increases risk of metabolic syndrome, type-2 diabetes and its components. Various previous studies evaluated that oxidative stress is associated with metabolic syndrome, type-2 diabetes and its components. Mustafa zbek et al. has showed that oxidative stress (OX) is known to play a major role in the pathogenesis of metabolic syndrome and its components. Paul Holvoet et al. (2004); showed that metabolic syndrome is associated with a higher fraction of circulatory OxLDL and thus with higher levels of circulating OxLDL increases the risk of metabolic syndrome, type-2 diabetes and its components [16]. The results of present study levels for circulatory oxidized low-density lipoprotein (OxLDL) suggests that higher concentration of circulatory oxidized low-density lipoprotein (OxLDL) was significantly associated with metabolic syndrome, type-2 diabetes and type- 2 diabetes with hypertension when compared to normal subjects. We have also observed that the mean circulatory OxLDL levels among diabetes alone and diabetes with hypertension was also found to be statistically significant. This observation was consistent with previous results analysis of (Koubaa et al. 2007); which suggests that higher OxLDL level was detected in type- 2 diabetes patients with hypertension as compared with diabetic patients without hypertension [17].

\section{Conclusion}

It is concluded that OxLDL may serves as better predictive biomarker in metabolic syndrome individuals. Further, extensive and elaborated research will validate its use to diagnose subjects with metabolic syndrome, diabetes with or without hypertension when compared to internationally defined criteria to ultimately provide cost effective tools for metabolic syndrome diagnosis. Moreover, further extensive studies are required to show the association between oxidative stress markers in type- 2 diabetes mellitus, type- 2 diabetes mellitus with hypertension and metabolic syndrome.

\section{Conflict of Interests}

Authors have declared that no conflict of interests exists.

\section{Ethics Approval and Consent to Participate}

This study was approved by institutional ethical committee King George's Medical University, Lucknow. All subject signed the approved informed consent. (Ethical clearence no :71 ECM II B Thesis/P 13).

\section{References}

1. Expert Panel on Detection E (2001) Executive summary of the Third Report of the National Cholesterol Education Program (NCEP) expert panel on detection, evaluation, and treatment of high blood cholesterol in adults (Adult Treatment Panel III). Jama 285: 2486.

2. Oda E (2006) Criteria for diagnosing the metabolic syndrome. Author's reply. The 84: 1251-1252.

3. Grundy SM, Cleeman JI, Daniels SR, Donato KA, Eckel RH, et al. (2005) Diagnosis and Management of the Metabolic Syndrome: An American Heart Association/National Heart, Lung, and Blood Institute Scientific Statement. Circulation 112: 2735-2752.

4. Alberti K, Eckel R, Grundy S, Zimmet P, Cleeman J, et al. (2009) Harmonizing the Metabolic Syndrome. Circulation 16: 1640-1645.

5. Primeau V, Coderre L, Karelis AD, Brochu M, Lavoie ME, Messier V, et al. (2011) Characterizing the profile of obese patients who are metabolically healthy. Int Obesity 35: 971-981.

6. Cipolletta C, Ryan KE, Hanna EV, Trimble ER (2005) Activation of peripheral blood CD14+ monocytes occurs in diabetes. Diabetes 54 : 2779-2786.

7. Osman MT, Rahman T, Ismail TS, Azlina AR, Nawawi H (2016) Investigation of Oxidative Stress Status in Metabolic Syndrome Patients Using Lipid Peroxidation Biomarkers. Int Arch Med. 4: 9.

8. Betteridge DJ (2000) What is the oxidative stress in. Metabolism 4913-4918.

9. Montuschi P, Barnes PJ, Roberts LJ (2004) Isoprostanes: markers and mediators of oxidative stress. The FASEB journal 18: 1791-1800.

10. Holvoet P, Lee DH, Steffes M, Gross M, Jacobs DR (2008) Association Between Circulating Oxidized Low-Density Lipoprotein and Incidence of the Metabolic Syndrome. JAMA 299: 2287-2293.

11. Antonopoulos S (2002) Third Report of the National Cholesterol Education Program (NCEP) Expert Panel on Detection, Evaluation, and Treatment of High Blood Cholesterol in Adults (Adult Treatment Panel III) final report. Circulation 106: 3143-3421.

12. Grundy SM, Bilheimer D, Chait A, Clark LT, Denke M, et al. (1993) Summary of the second report of the National Cholesterol Education Program (NCEP) Expert Panel on detection, evaluation, and treatment of high blood cholesterol in adults (Adult Treatment Panel II). Jama 269: 3015-3023.

13. Kawamoto R, Tabara Y, Kohara K, Miki T, Kusunoki T, et al. (2011) Relationships between lipid profiles and metabolic syndrome, insulin resistance and serum high molecular adiponectin in Japanese community-dwelling adults. Lipids in Health and Disease 10: 79.

14. Lewis GF, Uffelman KD, Szeto LW, Steiner G (1993) Effects of acute hyperinsulinemia on VLDL triglyceride and VLDL ApoB production in normal weight and obese individuals. Diabetes 42: 833-842.

15. Kwon YS (2011) Metabolic syndrome components and living habits according to fasting blood sugar measured by glucometer in workers. Indian J Sci Tech 8: 37-45.

16. Holvoet P, Kritchevsky SB, Tracy RP, Mertens A, Rubin SM, et al. (2004) The metabolic syndrome, circulating oxidized LDL, and risk of myocardial infarction in well-functioning elderly people in the Health, Aging, and body composition cohort. Diabetes 53: 1068-1073.

17. Koubaa N, Nakbi A, Smaoui M, Abid N, Chaaba R, et al. (2007) Hyperhomocysteinemia and elevated ox-LDL in Tunisian type 2 diabetic patients: Role of genetic and dietary factors. Clin Biochem 13:1007-1014. 